\title{
Thermal Instability in a Porous Medium with Random Vibrations
}

\author{
By
}

\author{
B. S. Dandapat and A. S. Gupta, Kharagpur, India \\ With 4 Figures
}

(Received November 28, 1980; revised January 26, 1981)

\begin{abstract}
Summary
Onset of thermal convection in a layer of saturated porous medium, heated from below, is examined when the layer is subjected to random vibrations. It is shown that when the vibrations are characterized by a white noise process, they hasten the onset of convection. Further, decrease in permeability tends to stabilize the flow field.
\end{abstract}

\section{Introduction}

Several experiments were conducted by Morrison [1], Morrison, Rogers and Horton [2], and Rogers and Schilberg [3] for observing the onset of convection in a horizontal layer of a saturated porous medium heated from below. The observed critical temperature gradient was smaller by an order of magnitude than the gradient predicted from the theoretical investigations of Horton and Rogers [4], and Lapwood [5]. Extension of these theoretical studies was made by Wooding [6], [7]. The quantitative disagreement between the theory and experiments was sought to be removed by Rogers and Morrison [8], Morrison and Rogers [9], and Rogers [10] by allowing for the temperature dependence of viscosity, an initially steady nonlinear temperature distribution, and a columnar rather than cellular form of convection. However the experiments mentioned above involved a timedependent situation and so cannot be regarded as a satisfactory test of the Lapwood theory which assumes an initially steady temperature distribution. Elder [11] and Combarnous and Le Fur [12] determined experimentally the point at which thermal convection began and found good agreement with the theory. An extension of Lapwood's theory was made by Gheorghitza [13] who assumed the porous medium to be nonhomogeneous. The global stability of convective flow in a porous medium using energy method was studied by Westbook [14]. Dependence of Nusselt number on the Rayleigh number in steady convection in a porous medium was investigated by Palm, Weber and Kvernvold [15]. Using a variational method, the bounds on heat transport in a porous medium were determined by Busse and Joseph [16]. Strauss and Schubert [17] and Horne [18] studied twodimensional and three-dimensional natural convection in a confined porous medium heated from below. 
In all the above investigations the forces acting on the system are deterministic. In this paper we study the thermal instability in a layer of saturated porous medium which is heated from below and is subjected to random vibrations. In particular the vibrations are characterized by a white noise process and their effects on the onset of convection are investigated. The motivation for this study comes doubtless from geophysics with particular reference to the influence of earthquakes on convective instabilities in the upper mantle of the earth. It is therefore meaningful to consider the onset of convection in such a geothermal region (which is a porous medium) subjected to random vibrations. Of course in the case of earthquake waves, definite frequencies are preferred and modelling such waves by a stochastic process of white noise type would certainly be an oversimplification of a complex process. Nevertheless we feel that the study of thermal convection using a white noise model would perhaps be a first step towards understanding the effect of earthquakes on thermal convection. The present study has also some methodological interest since the stability analysis is reduced to the study of a certain class of stochastic (random) differential equations (Soong [19]). We shall, however, confine ourselves to the mean square stability of the solution of such equations. The physical significance of a white noise process and its limitations are discussed in Section 2.

\section{Mathematical Formulation and Stability Analysis}

Consider a layer of saturated porous medium of thickness $d$ between the planes $z=0$ and $z=d$, $z$-axis being vertically upward. The layer is infinite in the horizontal direction and is heated from below leading to a uniform adverse temperature gradient $\beta_{1}=\left(T_{1}-T_{2}\right) / d$, where $T_{1}$ and $T_{2}$ are the constant temperatures of the lower and upper surface respectively with $T_{1}>T_{2}$. Further we assume that the porous layer oscillates vertically in a random fashion so that the total gravitational acceleration is $g+g^{\prime}(t)$, where $g$ is the mean gravity and $g^{\prime}(t)$ is a stochastic process. Fig. 1 gives a sketch of the physical problem with the used symbols.

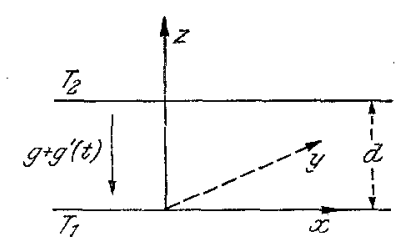

Fig. 1. Sketch of the physical problem

The general form of the Darcy law governing the motion of an incompressible fluid through a homogeneous isotropic porous medium is given by (Nield [20])

$$
\frac{\varrho}{\varepsilon} \frac{D \boldsymbol{q}}{D t}=-\nabla p-\frac{\mu}{K} \boldsymbol{q}+\mu \nabla^{2} \boldsymbol{q}+\varrho \boldsymbol{X}
$$

where $\varrho, \varepsilon, \boldsymbol{q}, p, \mu, K$ and $\boldsymbol{X}$ denote fluid density, porosity of the medium, filtration velocity, fluid pressure, dynamic viscosity coefficient, permeability and body 
force per unit mass respectively. For very fluffy foam metal materials or fibrous materials, $\varepsilon$ is very close to unity and in beds of packed spheres, $\varepsilon$ is in the range of $0.25-0.50$ (Joseph [21]). Although the viscous term $\mu \nabla^{2} q$ is generally neglected in slow motion through a porous medium, it should be taken into account for the general flow particularly in the case $\varepsilon \approx 1$, when the fluid occupies all parts of the porous medium. The equation of continuity is

$$
\boldsymbol{V} \cdot \boldsymbol{q}=0
$$

while the energy equation is (see Caltagirone [22])

$$
(\varrho c)^{*} \frac{\partial T}{\partial t}+(\varrho c)_{f} \boldsymbol{q} \cdot \nabla T=\lambda^{*} \nabla^{2} T
$$

The porous medium formed by the porous matrix and the interstitial fluid (which is the fluid in the pores) is regarded as a fictitious isotropic fluid with heat capacity $(\varrho c)^{*}=\varepsilon(\varrho c)_{f}+(1-\varepsilon)(\varrho c)_{s}$, where $(\varrho c)_{f}$ and $(\varrho c)_{s}$ denote the heat capacity of the fluid and the solid respectively. The physical properties of the medium with an effective thermal conductivity $\lambda^{*}$ are assumed constant, in particular with respect to temperature dependence. The variations in density with temperature are neglected in the present analysis, except with regard to their influence on the buoyancy force (Boussinesq approximation).

Within the framework of linear stability theory, the equations of momentum, mass and energy for infinitesimal perturbations imposed on the quiescent initial state (the frame of reference is fixed in the porous layer) are given by (1), (2) and (3) as

$$
\begin{gathered}
\frac{1}{\varepsilon} \frac{\partial u}{\partial t}=-\frac{1}{\varrho_{0}} \frac{\partial p}{\partial x}-\frac{\nu}{K} u+\nu \nabla^{2} u \\
\frac{1}{\varepsilon} \frac{\partial v}{\partial t}=-\frac{1}{\varrho_{0}} \frac{\partial p}{\partial y}-\frac{\nu}{K} v+\nu \nabla^{2} v \\
\frac{1}{\varepsilon} \frac{\partial w}{\partial t}=-\frac{1}{\varrho_{0}} \frac{\partial p}{\partial z^{v}}-\frac{\nu}{K} w+\left(g+g^{\prime}\right) \alpha \theta+\nu \nabla^{2} w \\
\frac{\partial u}{\partial x}+\frac{\partial v}{\partial y}+\frac{\partial w}{\partial z}=0 \\
\frac{\partial \theta}{\partial t}+\beta w=K_{1} \nabla^{2} \theta
\end{gathered}
$$

where

$$
\begin{gathered}
\beta=\beta_{1}(\varrho c)_{f} /(\varrho c)^{*}, \quad T=T_{1}+\beta_{1} z+\theta, \quad v=\mu / \varrho_{0}, \\
\boldsymbol{q} \equiv(u, v, w), \quad K_{1}=\lambda^{*} /(\varrho c)^{*} .
\end{gathered}
$$

In writing the above equations, the equation of state $\varrho=\varrho_{0}\left[1-\alpha\left(T-T_{0}\right)\right]$ is used where $\alpha$ is the coefficient of volume expansion and $\varrho_{0}$ and $T_{0}$ denote density and temperature of a reference state.

Eliminating all the variables in favor of the dimensionless vertical velocity $w_{1}(=w d / v)$ and the dimensionless temperature perturbation $\theta_{1}(=\theta / \beta d)$, we 
obtain from (4)-(8) the following dimensionless equations:

$$
\begin{gathered}
\frac{1}{\varepsilon} \frac{\partial}{\partial \tau} \nabla^{2} w_{1}=\frac{g \alpha \beta d^{4}}{v^{2}}[1+G(\tau)] \nabla_{1}{ }^{2} \theta_{1}-B \nabla^{2} w_{1}+\nabla^{4} w_{1}, \\
\operatorname{Pr}\left(\frac{\partial \theta_{1}}{\partial \tau}+w_{1}\right)=\nabla^{2} \theta_{1} .
\end{gathered}
$$

Here

$$
\begin{gathered}
\tau=\frac{v t}{d^{2}}, \quad(X, Y, Z)=(x / d, y / d, z / d), \nabla_{1}^{2}=\partial^{2} / \partial X^{2}+\partial^{2} / \partial Y^{2}, \\
\operatorname{Pr}=v / K_{1}, \nabla^{2}=\partial^{2} / \partial X^{2}+\partial^{2} / \partial Y^{2}+\partial^{2} / \partial Z^{2}, B=d^{2} / K, \\
G(\tau)=g^{\prime}(t) / g .
\end{gathered}
$$

We next use normal mode technique to solve (10) and (11). When $w_{1}$ is eliminated from (10) and (11) and $\theta_{1}$ is taken as

$$
\theta_{\mathbf{1}}=A(\tau) \sin \pi Z \exp \left[i\left(k_{x} X+k_{y} Y\right)\right]
$$

in the resulting equation, the following equation for the amplitude $A(\tau)$ is obtained

where

$$
\frac{d^{2} A}{d \tau^{2}}+C_{1} \frac{d A}{d \tau}+D_{1} A=\frac{a^{2} R G(\tau) \varepsilon}{\operatorname{Pr} \gamma^{2}} A,
$$

$$
\begin{gathered}
a^{2}=k_{x}{ }^{2}+k_{y}{ }^{2}, \quad \gamma^{2}=\pi^{2}+a^{2}, \quad R=-g \alpha \beta d^{4} / \nu K_{1}, \\
C_{1}=\left(\gamma^{2} / \operatorname{Pr}\right)+\left(B+\gamma^{2}\right) \varepsilon, \quad D_{1}=\varepsilon\left[\gamma^{4}\left(B+\gamma^{2}\right)-\operatorname{Ra}^{2}\right] / \operatorname{Pr} \gamma^{2} .
\end{gathered}
$$

It may be seen from (14) that in addition to the time-dependent stochastic parameter $G(\tau)$, the stability characteristics depend on the Rayleigh number $R$, the Prandtl number $\operatorname{Pr}$, the permeability parameter $B$ and the porosity $\varepsilon$. Note that the expression (13) for $\theta_{1}$ corresponds to the lowest mode and is consistent with our assumption of two free surfaces at $Z=0$ and $Z=1$. At these two surfaces the vertical velocity component $w_{1}$, the temperature perturbation $\theta_{1}$ and the shear stress must vanish so that

$$
\theta_{1}=\partial^{2} \theta_{1} / \partial Z^{2}=\cdots=\partial^{2 n} \theta_{1} / \partial Z^{2 n} \quad(n=2,3, \ldots) \text { at } Z=0 \text { and } 1
$$

upon using (10) and (11). It may be remarked that the choice of free boundaries is not so artificial since the porous problem can accomodate permeable walls.

Instead of attempting to find the explicit solution of (14), we shall seek the mean square stability criterion for this solution. This implies that every mean square bounded input leads to a mean square bounded output. To this end we assume that $G(\tau)$ is given by a white noise process (Soong [19]) and recast (14) as a two-dimensional Ito equation given by

$$
d \tilde{A}(\tau)=\tilde{f}(\tilde{A}(\tau), \tau) d \tau+H(\tilde{A}(\tau), \tau) d \tilde{B},
$$

where the vectors $\tilde{A}, \tilde{f}$ and the matrix $H$ are as follows:

$$
\tilde{A}=\left[\begin{array}{l}
A_{1} \\
A_{2}
\end{array}\right], \quad \tilde{f}=\left[\begin{array}{l}
A_{2} \\
-D_{1} A_{1}-C_{1} A_{2}
\end{array}\right], \quad H=\left[\begin{array}{ll}
0 & 0 \\
\frac{\operatorname{Ra}^{2} \varepsilon A_{1}}{\operatorname{Pr} \gamma^{2}} & 0
\end{array}\right]
$$


with $A_{1}=A(\tau)$ and $A_{2}=d A / d \tau$. The reason for choosing white noise process (which is an abstraction or a limiting process) is two-fold. First, this is one of the most commonly used stochastic processes which are amenable to a fairly simple and straightforward mathematical analysis. Hence we use it as an approximation to a number of random physical phenomena. Secondly, the use of the white noise models simulates well the behaviour of the actual stochastic process if one deals with a small portion of the frequency spectrum in the analysis (Soong [19]). The term 'white' is introduced in connection with the white light, which has the property that its power spectral density is flat over the visible portion of the electromagnetic spectrum. However it is customary to define a white noise in a stronger sense so that its power spectral density is constant for all frequencies. The vector $\tilde{B}(\tau), \tau \geqq 0$ defined in (17) represents a two-dimensional Brownian motion process (or a Wiener process) with zero mean such that

$$
\frac{d \tilde{B}}{d \tau}=\left[\begin{array}{l}
G(\tau) \\
0
\end{array}\right]
$$

It should be noted that the stochastic process $\tilde{B}(\tau)$ is not mean square differentiable so that in (19) we consider the derivative in the formal sense. Clearly $d \tilde{B} / d \tau$ is Gaussian with mean zero.

If $h(\tilde{A}(\tau), \tau)$ is an arbitrary function of $\tilde{A}(\tau)$ and $\tau$, the following moment equation can be derived from (17) (Soong [19]) as

$$
\begin{aligned}
\frac{d}{d \tau} E\{h(\tilde{A}(\tau), \tau)\}= & \sum_{j=1}^{2} E\left\{f_{j} \frac{\partial h}{\partial A_{i}}\right\} \\
& +\sum_{i, j=1}^{2} E\left\{\left(H D H^{T}\right)_{i j} \frac{\partial^{2} h}{\partial A_{i} \partial A_{j}}\right\}+E\left\{\frac{\partial h}{\partial \tau}\right\},
\end{aligned}
$$

where $E$ stands for mathematical expectation and $D$ stands for the $2 \times 2$ matrix with elements $D_{i j}$ satisfying

$$
E\left[\Delta B_{i}(\tau) \Delta B_{j}(\tau)\right]=2 D_{i j} \Delta \tau, \quad i, j=1,2 .
$$

Here $\Delta B_{i}(\tau)\left(=B_{i}(\tau+\Delta \tau)-B_{i}(\tau)\right)$ is an inerement in $B_{i}(\tau)$ during time interval $\Delta \tau$.

Introducing the moment $\Delta$

$$
m_{j k}(\tau)=E\left\{A_{1}{ }^{j}(\tau) A_{2}^{k}(\tau)\right\},
$$

we find from (20) the first-order moment equations as

$$
\dot{m}_{10}(\tau)=m_{01}(\tau), \quad \dot{m}_{01}(\tau)=-D_{1} m_{10}(\tau)-C_{1} m_{01}(\tau)
$$

where an overdot denotes derivative with respect to $\tau$. Similarly the second-order moment equations are

$$
\begin{aligned}
& \dot{m}_{20}=2 m_{11}, \quad \dot{m}_{11}=-D_{1} m_{20}-C_{1} m_{11}+m_{02}, \\
& \dot{m}_{02}=2\left[D_{11}\left(\frac{\varepsilon \mathrm{Ra}^{2}}{\operatorname{Pr} \gamma^{2}}\right)^{2} m_{20}-D_{1} m_{11}-C_{1} m_{02}\right],
\end{aligned}
$$


where $D_{11}$ is the only nonzero element in $D_{i j}$ for a white noise process. As these equations are linear, the solutions are assumed in the form

$$
m_{10}(\tau)=\sum_{j=1}^{2} \alpha_{j} \exp \left(\lambda_{j} t\right) ; \quad m_{01}(\tau)=\sum_{j=1}^{2} \beta_{j} \exp \left(\lambda_{j} t\right) .
$$

In order that the first-order moments be asymptotically stable, the real parts of $\lambda_{i}$ must be negative. Substituting (25) in (23) we find that for nontrivial solutions, $\lambda_{j}$ must satisfy

$$
\lambda^{2}+C_{1} \lambda+D_{1}=0
$$

In order that its roots have negative real parts, we must have

$$
C_{1}>0 \text { and } D_{1}>0 \text {. }
$$

These are the requirements for asymptotic stability in the mean.

The criterion for asymptotic mean square stability can be obtained from (24) in the same manner. The characteristic equation in this case is

$$
\left|\begin{array}{ccc}
\lambda & -2 & 0 \\
D_{1} & C_{1}+\lambda & -1 \\
-2 D_{11}\left(\frac{\varepsilon \operatorname{Ra}^{2}}{\operatorname{Pr} \gamma^{2}}\right)^{2} 2 D_{1} & 2 C_{1}+\lambda
\end{array}\right|=0
$$

which on simplification reduces to

$$
\lambda^{3}+3 C_{1} \lambda^{2}+2\left(C_{1}^{2}+2 D_{1}\right) \lambda+4\left(C_{1} D_{1}-\frac{D_{11} \varepsilon^{2} R^{2} a^{4}}{\operatorname{Pr}^{2} \gamma^{4}}\right)=0
$$

Using the Routh-Hurwitz criterion, it can be shown that the second-order moments are stable if all the coefficients in (28) are positive and the inequality

$$
6 C_{1}\left(C_{1}^{2}+2 D_{1}\right)>4\left(C_{1} D_{1}-\frac{D_{11} \varepsilon^{2} R^{2} \alpha^{4}}{\operatorname{Pr}^{2} \gamma^{4}}\right)
$$

holds. The coefficients in (28) will be positive if

$$
\begin{gathered}
\left(\gamma^{2} / \operatorname{Pr}\right)+\left(B+\gamma^{2}\right) \varepsilon>0 \\
{\left[\frac{\gamma^{2}}{\operatorname{Pr}}+\left(B+\gamma^{2}\right) \varepsilon\right]^{2}+\frac{2 \varepsilon\left[\gamma^{4}\left(B+\gamma^{2}\right)-\mathrm{Ra}^{2}\right]}{\operatorname{Pr}^{2} \gamma^{2}}>0} \\
{\left[\frac{\gamma^{2}}{\operatorname{Pr}}+\left(B+\gamma^{2}\right) \varepsilon\right]\left[\frac{\gamma^{4}\left(B+\gamma^{2}\right)-\mathrm{Ra}^{2}}{\operatorname{Pr} \gamma^{2}}\right]-\frac{D_{11} \varepsilon R^{2} a^{4}}{\operatorname{Pr}^{2} \gamma^{4}}>0}
\end{gathered}
$$

after recalling the definitions of $C_{1}$ and $D_{1}$ from (15).

Condition (30) is always satisfied. Inequality (31) is satisfied when

$$
R<\frac{\gamma^{4}\left(B+\gamma^{2}\right)}{a^{2}}+\frac{\gamma^{2}\left[\gamma^{2}+\left(B+\gamma^{2}\right) \varepsilon \operatorname{Pr}\right]^{2}}{2 \varepsilon a^{2} \operatorname{Pr}}
$$

This implies a stabilizing effect whose magnitude depends on $\operatorname{Pr}, \varepsilon$ and $B$ but not on the stochastic parameter $D_{11}$. The inequality (32) ensures that the right hand 
side of (29) is positive. Since the Routh-Hurwitz criterion provides both necessary and sufficient conditions for stability, violation of (32) alone would imply instability even if the remaining inequalities are satisfied. For given values of $\operatorname{Pr}$, $D_{11}, B$ and $\varepsilon$, the regions of stability and instability in the $(a-R)$ plane are separated by the curve whose equation is any one of the following relationships:

$$
\begin{aligned}
R_{1,2}= & \frac{1}{2 D_{11} \varepsilon a^{4}}\left[-a^{2} \gamma^{2}\left\{\gamma^{2}+\left(B+\gamma^{2}\right) \varepsilon \operatorname{Pr}\right\} \pm\left\{a^{4} \gamma^{4}\left[\gamma^{2}+\left(B+\gamma^{2}\right) \varepsilon \operatorname{Pr}\right]^{2}\right.\right. \\
& \left.\left.+4 \gamma^{6}\left(B+\gamma^{2}\right)\left(\gamma^{2}+\left(B+\gamma^{2}\right) \varepsilon \operatorname{Pr}\right) D_{11} \varepsilon a^{4}\right\}^{1 / 2}\right]
\end{aligned}
$$

It may be seen that the position of the stability boundary depends on the random parameter $D_{11}$.

It is also clear that of the two values $R_{1,2}$, the one which gives positive Rayleigh number $\left(R_{1}\right.$, say) will be relevant to our discussion. The critical Rayleigh number $R^{*}$ at the onset of convection will correspond to the minimum of $R_{1}$ with respect to the wave number $\alpha$ so that $d R_{1} / d \alpha=0$ at $R_{1}=R^{*}$. Using this fact in (34), the expression for the critical Rayleigh number is given by

$$
\begin{aligned}
R^{*}=\frac{1}{2 D_{11} \varepsilon s^{2}}[ & -\left(\pi^{2} s+s^{2}\right)\left\{\pi^{2}+s+\left(B+\pi^{2}+s\right) \varepsilon \operatorname{Pr}\right\} \\
& +\left\{\left(\pi^{2} s+s^{2}\right)^{2}\left[\pi^{2}+s+\left(B+\pi^{2}+s\right) \varepsilon \operatorname{Pr}\right]^{2}\right. \\
& +4\left(\pi^{2}+s\right)^{3}\left(B s^{2}+\pi^{2} s^{2}+s^{3}\right) \\
& \left.\left.\times\left(\pi^{2}+s+\left(B+\pi^{2}+s\right) \varepsilon \operatorname{Pr}\right) D_{11} \varepsilon\right\}^{1 / 2}\right]
\end{aligned}
$$

where $s$ is a positive root of the following equation:

$$
\begin{aligned}
& 2\left[-\left(\pi^{2} s+s^{2}\right)\left(A_{1}^{*}+C_{1}{ }^{*} s\right)+\left\{\left(\pi^{2} s+s^{2}\right)^{2}\left(A_{1}{ }^{*}+C_{1} *\right)^{2}\right.\right. \\
& \left.\left.+4 D_{11} \varepsilon\left(\pi^{2}+s\right)^{3}\left(B_{1}^{*}+s\right)\left(A_{1}^{*} s^{2}+C_{1} *^{3}\right)\right\}^{1 / 2}\right] \\
& =-\left\{\pi^{2} A_{1}^{*} s+2\left(A_{1}{ }^{*}+\pi^{2} C_{1}^{*}\right) s^{2}+3 C_{1}{ }^{*} s^{3}\right\}+\left\{\left(\pi^{2} s+s^{2}\right)^{2}\left(A_{1}^{*}+C_{1}^{*} s\right)^{2}\right. \\
& \left.+4 D_{11} \varepsilon\left(\pi^{2}+s\right)\left(B_{1}^{*}+s\right)\left(A_{1} * s^{2}+C_{1} s^{3}\right)\right\}^{-1 / 2} \cdot\left\{\pi^{4} A_{1}^{* 2}+4 \pi^{6} A_{1}^{*} B_{1} * D_{11} \varepsilon\right) s^{2} \\
& +\left(3 \pi^{2} A_{1}{ }^{* 2}+3 \pi^{4} A_{1} * C_{1}{ }^{*}+18 \pi^{4} A_{1} * B_{1}{ }^{*} D_{11} \varepsilon+6 \pi^{6} A_{1} * D_{11} \varepsilon+6 \pi^{6} B_{1}{ }^{*} C_{1}{ }^{*} D_{11} \varepsilon\right) s^{3} \\
& +\left(4 \pi^{2} A_{1}{ }^{*} C_{1}{ }^{*}+2\left(A_{1}{ }^{*}+\pi^{2} C_{1}{ }^{*}\right)^{2}+8 D_{11} \varepsilon\left(3 \pi^{2} A_{1}{ }^{*} B_{1}{ }^{*}+3 \pi^{4} A_{1}{ }^{*}+3 \pi^{4} B_{1}{ }^{*} C_{1} *\right.\right. \\
& \left.\left.+\pi^{6} C_{1}{ }^{*}\right)\right) s^{4}+\left(5 A_{1}{ }^{*} C_{1}{ }^{*}+5 \pi^{2} C_{1}{ }^{*}+10 D_{11} \varepsilon\left(A_{1}{ }^{*} B_{1}{ }^{*}+3 \pi^{2} A_{1}{ }^{*}+3 \pi^{2} B_{1}{ }^{*} C_{1} *\right.\right. \\
& \left.\left.\left.+3 \pi^{4} C_{1}^{*}\right)\right) s^{5}+\left(3 C_{1}^{* 2}+12 D_{11} \varepsilon\left(A_{1}^{*}+B_{1}^{*} C_{1}^{*}+3 \pi^{2} C_{1}^{*}\right)\right) s^{6}+14 C_{1}^{*} D_{11} \varepsilon s^{7}\right\}
\end{aligned}
$$

with

$$
A_{1}^{*}=\pi^{2}+\left(B+\pi^{2}\right) \varepsilon \operatorname{Pr}, \quad B_{1}^{*}=\pi^{2}+B, \quad C_{1}^{*}=1+\varepsilon \operatorname{Pr} .
$$

However the evaluation of $R^{*}$ from (35) by solving the highly complicated Eq. (36) poses insuperable difficulties. We have circumvented this problem by computing $R_{1}$ versus $a^{2}$ from (34) for several values of $\varepsilon, B$ and $D_{11}$ with $\operatorname{Pr}=7$ (water). It is found that $R_{1}$ has a minimum $\left(R^{*}\right)$ for a definite value of $a$ when the other parameters are fixed. 


\section{Results and Discussion}

Fig. 2 shows the variation of $R_{1}$ with $\alpha^{2}$ for several values of $B$ with $\varepsilon=0.6$ and $D_{11}=1$. Each curve has a minimum (which gives $R^{*}$ ) and $R^{*}$ increases with $B$. Since $B=d^{2} / K$, the implication of this result is that a decrease in permeability (for a fixed value of $d$ ) tends to stabilize the flow fields. Fig. 3 shows the variation of $R_{1}$ with $a^{2}$ for several values of $\varepsilon$ with $D_{11}=1$ and $B=1$. It can be seen that the critical Rayleigh number decreases with increase in $\varepsilon$. The interpretation of this result is that the Rayleigh-Bénard system is a lower bound and decreasing porosity tends to exert a stabilizing influence on the flow.

Fig. 4 displays the plot of $R_{1}$ versus $\alpha^{2}$ for several values of the random parameter $D_{11}$ with $\varepsilon=0.6$ and $B=1$. It is clear that $R^{*}$ steadily decreases with increase in $D_{11}$ and this shows that random vibrations tend to destabilize the flow. This can also be shown analytically from (34) since $\partial R_{1} / \partial D_{11}$ is always negative for given values of $a^{2}, B, \varepsilon$ and Pr. As $D_{11} \rightarrow 0$, it follows from (34) on binomial expansion in powers of $D_{11}$ that

$$
R_{1}=\gamma^{4}\left(B+\gamma^{2}\right) / a^{2} .
$$

It is interesting to note that in this limit, $R_{1}$ depends neither on $\operatorname{Pr}$ nor on $\varepsilon$. Further when $B=0$, we retrieve from (38) the classical result in Bénard convection in the absence of a porous medium as $R_{1}=\gamma^{6} / a^{2}$ (Chandrasekhar [23]). The absence of $\varepsilon$ in (38) in the limit $D_{11} \rightarrow 0$ may be explained physically as follows. The porosity factor $\varepsilon$ appears only in the convective term of the momentum equation. In the absence of random vibrations, the present problem reduces to the Bénard problem where a stationary marginal state prevails (Lapwood [5]). This shows that there is no effect of porosity on thermal convection unless random disturbances are examined, in which case its effect is that of a scaling factor for $D_{11}$. Note that in the foregoing analysis, the stochastic component of gravity $g^{\prime}$ is not assumed small. This means that the above stability analysis is valid for any value of $D_{11}$ characterizing the white noise process. To see what size of random variation in gravity will produce a significant lowering of the Rayleigh number, we proceed as follows. In the absence of random vibrations, the critical Rayleigh number $R^{*}$ computed from (38) for $B=1$ is 701.69 and this value occurs at $x^{2}=5.09$. On the other hand, Fig. 4 shows that for $B=1, R^{*} \approx 200$ when $D_{11}=5.0$. Thus we see that $D_{11}=0(5)$ causes a significant lowering of the critical Rayleigh number. Similar calculations can be performed in any practical situation as there is no restriction on the value of $D_{11}$. We are not, however, aware of any actual data in this respect but our guess is that in any natural random forcing, much lower value of $D_{11}$ would initiate convection.

The eigen functions in this problem can be found from (13) with amplitude $A(\tau)$ given by a stochastic process. When convection takes place slightly past the marginal state we might expect that this amplitude (in the mean square sense) will vary as $\left(R-R^{*}\right)^{1 / 2}$ (Chandrasekhar [23]). Since the behavior of $R^{*}$ for various values of $\varepsilon, B$ and $D_{11}$ is known from Figs. 2,3 and 4 , we can compute the eigen functions for special value of any of these parameters. For example we may say that the amplitude in the eigen function (13) increases with increase in the 


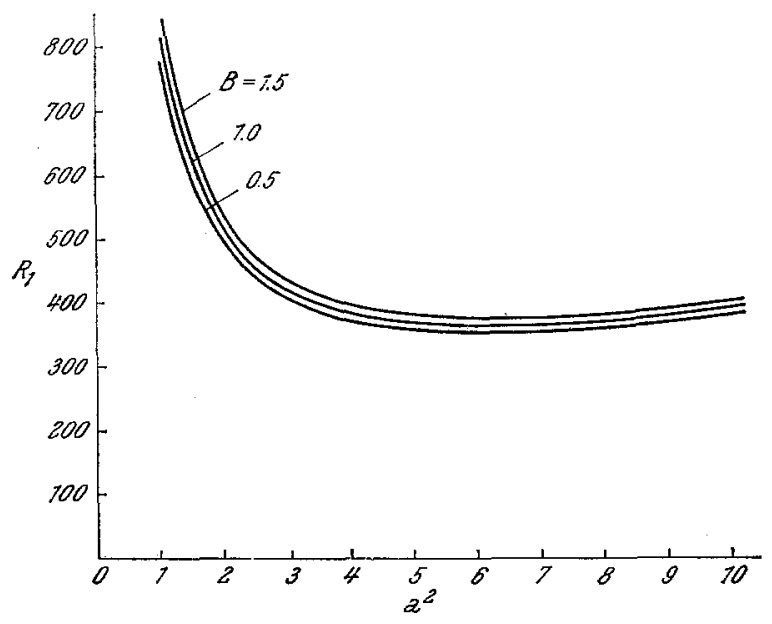

Fig. 2. Variation of $R_{1}$ versus $a^{2}$ for several values of $B$ with $\varepsilon=0.6, D_{11}=1$ and $\operatorname{Pr}=7$

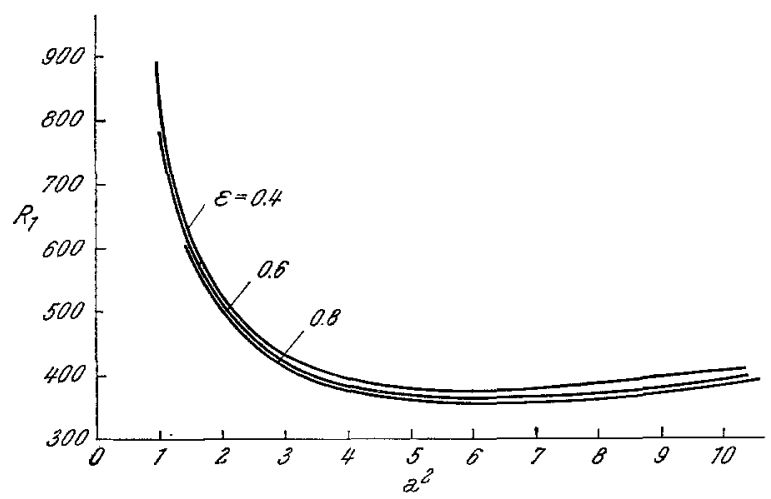

Fig. 3. Variation of $R_{1}$ versus $a^{2}$ for several values of $\varepsilon$ with $B=1, D_{11}=1$ and $\operatorname{Pr}=7$

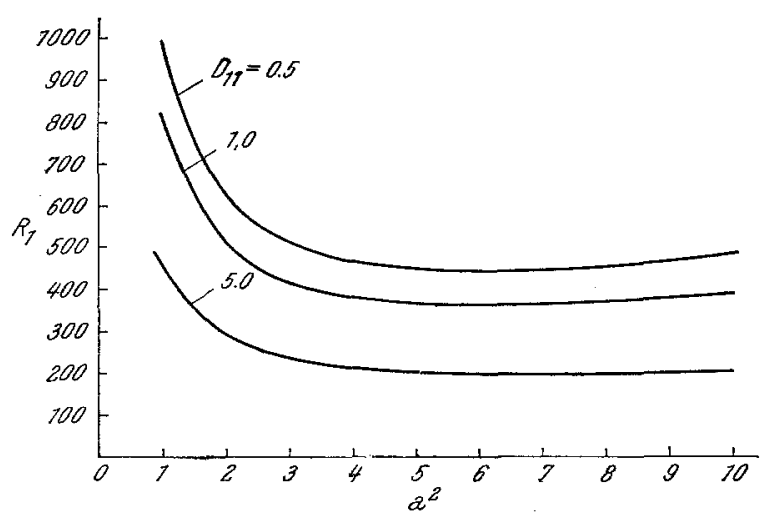

Fig. 4. Variation of $R_{1}$ versus $a^{2}$ for several values of $D_{11}$ with $\varepsilon=0.6, B=1$ and $\operatorname{Pr}=7$ 
stochastic parameter $D_{11}$ for fixed values of $\varepsilon$ and $B_{1}$ since $R^{*}$ decreases with increase in $D_{11}$.

We might offer a physical explanation of the destabilizing effects of random vibrations on convection as follows. Ignoring diffusive effects, a random gravitational forcing superimposed on the steady value, increases the mean square value of gravity and so makes disturbances to the system grow more rapidly. If, however, the fluctuating part has a carefully chosen frequency it may be possible to stabilize the system in a way similar to the inverted pendulum. Thus fixed (and hence deterministic) frequency oscillations may stabilize, but random ones will destabilize the system. As a matter of fact deterministic oscillations may either stabilize or destabilize a system depending on the amplitude and frequency of the imposed oscillations. In this connection mention may be made of the investigations of Yih and Li [24] who found that time-periodic modulation of the temperatures of the two plates has a destabilizing influence on the Bériard convection between the plates over a wide range of frequencies of modulation although the modulation is stabilizing at the low frequency end of the spectrum. Thus one would expect that in a thermal stability analysis based on a multichromatic disturbance (i.e. a disturbance averaged over all frequencies) of the plate temperature, modulation would have a net destabilizing influence since the stabilizing influence of modulation over the small part of the frequency spectrum would be overwhelmed by the destabilizing influence over a much wider range of frequencies. This prediction for deterministic modulations is, therefore, in keeping with our result of the destabilizing influence of random vibrations of the white noise type which may be regarded as a modulation averaged over all frequencies.

We thank Professor C.-S. Yih for some stimulating discussions on this paper.

\section{References}

[1] Morrison, H. L.: Preliminary measurements relative to the onset of thermal convection currents in unconsolidated sands. J. Appl. Physics 18, 849 (1947).

[2] Morrison, H. L., Rogers, Jr., F. T., Horton, C. W.: Convection currents in porous media. Observation of conditions at onset of convection. J. Appl. Physics 20, 1027 (1949).

[3] Rogers, Jr., F. T., Schilberg, L. E., Morrison, H. L.: Convection currents in porous media. Remarks on the theory. J. Appl. Physics 22, 1476 (1951).

[4] Horton, C. W., Rogers, Jr., F. T.: Convection currents in a porous medium. J. App]. Physics 16, 367 (1945).

[5] Lapwood, E. R.: Convection of a fluid in a porous medium. Proc. Camb. Phil. Soc. 44, 508 (1948).

[6] Wooding, R. A.: Steady state free thermal convection of liquid in a saturated permeable medium. J. Fluid Mech. 2, 273 (1957).

[7] Wooding, R. A.: An experiment on free thermal convection of water in saturated permeable material. J. Fluid Mech. 3, 582 (1958).

[8] Rogers, Jr., F. T., Morrison, H. L.: Convection currents in porous media. Extended theory of critical gradient. J. Appl. Physies 21, 1177 (1950).

[9] Morrison, H. L., Rogers, Jr., F.T.: Significance of flow-patterns for initial convection in porous media. J. Appl. Physies 23, 1058 (1952).

[10] Rogers, Jr., F. T.: Convection in porous media. Variational form of the theory. J. Appl. Physics 24, 877 (1953).

[11] Elder, J. W.: Steady free convection in a porous medium heated from below. J. Fluid Mech. 27, 29 (1967). 
[12] Combarnous, M., Lefur, B.: Transfert de chaleur par convection naturelle dans une couche poreuse horizontale. Comptes Rendus 269, 1009 (1969).

[13] Gheorghitza, S. I.: The marginal stability in porous inhomogeneous media. Proc. Camb. Phil. Soc. 57, 871 (1961).

[14] Westbrook, D. R.: Stability of convective flow in a porous medium. Phys. Fluids 12, $1547(1969)$.

[15] Palm, E., Weber, J. E., Kvernvold, O.: On steady convection in a porous medium. J. Fluid Mech. 54, 153 (1972).

[16] Busse, F. H., Joseph, D. D.: Bounds for heat transport in a porous layer. J. Fluid Mech. 54, 521 (1972).

[17] Strauss, J. M., Schubert, G.: Three-dimensional convection in a cubic box of fluidsaturated porous material. J. Fluid Mech. 91, 155 (1979).

[18] Horne, R. N.: Three-dimensional natural convection in a confined porous medium heated from below. J. Fluid Mech. 92, 751 (1979).

[19] Soong, T. T.: Random differential equations in science and engineering. Academic Press 1973.

[20] Nield, D. A.: Onset of thermohaline convection in a porous medium. Water Resources Research 4, 553 (1968).

[21] Joseph, D. D.: Stability of fluid motions IT. Berlin-Heidelberg-New York: Springer 1976.

[22] Caltagirone, J. P.: Thermoconvective instabilities in a porous medium bounded by two concentric horizontal cylinders. J. Fluid Mech. 76, 337 (1976).

[23] Chandrasekhar, S.: Hydrodynamic and hydromagnetic stability. Oxford University Press 1961.

[24] Yih, C.-S., Li, C.-H.: Instability of unsteady flows or configurations, part 2: Convective instability. J. Fluid Mech. 54, 143 (1972).

\author{
B. S. Dandapat and A. S. Gupta* \\ Mathematics Department \\ Indian Institute of Technology \\ Kharagpur \\ India
}

\author{
*Present address: \\ Department of Mech. Eng. and \\ Appl. Mech. College of Engineering \\ The University of Michigan \\ 550 East University \\ Ann Arbor, MI 48109, U.S.A.
}

